\title{
GRB 051221A and Tests of Lorentz Symmetry
}

\author{
María Rodríguez Martínez, Tsvi Piran and Yonatan Oren \\ Racah Institute of Physics, The Hebrew University, \\ 91904 Jerusalem, Israel.
}

\begin{abstract}
Various approaches to quantum gravity suggest the possibility of violation of Lorentz symmetry at very high energies. In these cases we expect a modification at low energies of the dispersion relation of photons that contains extra powers of the momentum suppressed by a high energy scale. These terms break boost invariance and can be tested even at relatively low energies. We use the light curves of the very bright short Gamma-Ray Burst GRB 051221A and compare the arrival times of photons at different energies with the expected time delay due to a modified dispersion relation. As no time delay was observed, we set a lower bound of $0.0066 E_{p l} \sim 0.66 \cdot 10^{17} \mathrm{GeV}$ on the scale of Lorentz invariance violation.
\end{abstract}

\section{Introduction}

Various quantum gravity theories suggest that Lorentz symmetry is broken or modified at very high energies [1. In most of these models the low energy limit of the photon dispersion relation is deformed by an addition of extra powers of the particle momentum. These modifications are suppressed by a high energy scale, beyond which Lorentz symmetry is broken. Among other implications, such terms cause the photon speed to depend on the energy and to differ from the classical speed of light, $c$ [2].

Amelino-Camelia et al. [3] proposed to use the arrival times of photons from cosmological Gamma Ray Bursts (GRBs) to set experimental bounds on the energy scale of Lorentz invariance violation. The idea was pursued by several groups which obtained observational bounds using ensembles of bursts with known redshfits 4 , 5, or using a single very powerful and atypical burst GRB 021206 [6]. In this paper we use the very bright short burst GRB 051221A to test Lorentz symmetry and set a new bound on the possible scale of Lorentz symmetry violation. 


\section{Lorentz Violation and Time Delay}

Following [7, we consider deformations of the dispersion relation of photons which break boost invariance but keep rotational and translational symmetry:

$$
E^{2}=\frac{p^{2} c^{2}}{a^{2}}\left[1+\left(\frac{p c}{\xi E_{p l} a}\right)^{n}\right], \quad n=1,2, \ldots .
$$

The non-standard term is suppressed by the high energy scale, $\xi E_{p l}$, where $\xi$ is a dimensionless parameter and $E_{p l}$ is the Planck energy. This expression can be thought as a low energy expansion (compared with $E_{p l}$ ) of a quantumgravity Hamiltonian. We will focus on $n=1$ and $n=2$ in the following.

Eq. 1 causes the speed of photons to depend on their energy. Two photons emitted simultaneously with different energies, arrive on Earth with a time delay $\Delta t_{\text {del }}$. By comparing this time delay (or the lack of it) with the time resolution of the observing detector we can set bounds on the Lorentz violation parameter, $\xi$. The time delay between two photons with energies $E_{1}$ and $E_{2}$ emitted simultaneously from a source at a redshift $z$ is given by [8]:

$\Delta t_{\mathrm{del}} \simeq \frac{1+n}{2 H_{0} \xi^{n}}\left[\left(\frac{E_{2}}{E_{p l}}\right)^{n}-\left(\frac{E_{1}}{E_{p l}}\right)^{n}\right] \frac{\sqrt{\Omega_{m}(1+z)^{3}+\Omega_{\Lambda}}}{\sqrt{\Omega_{m}+\Omega_{\Lambda}}} \int_{0}^{z} \frac{(1+z)^{n} d z}{\sqrt{\Omega_{m}(1+z)^{3}+\Omega_{\Lambda}}}$.

We use in the following the "standard" cosmological parameters $H_{0}, \Omega_{m}$ and $\Omega_{\Lambda}[9]$.

\section{GRB 051221A}

GRB 051221A was a very powerful bright short burst located in a star forming galaxy at a redshift of $z=0.5465$ [11. It was observed in the gamma ray regime by three different satellites: Swift-BAT, Suzaku-WAM and KonusWind. Swift-BAT and Konus-Wind have comparable instrumental time resolutions, $4 \mathrm{msec}$ and $2 \mathrm{msec}$ respectively. The time resolution of Suzaku-WAM is, on the other hand, significantly lower : $\sim 30$ msec. Since we need the highest time resolution for for our purposes [8], we only analyze the light curves of Swift-BAT and Konus-Wind which provide the strongest bounds. 


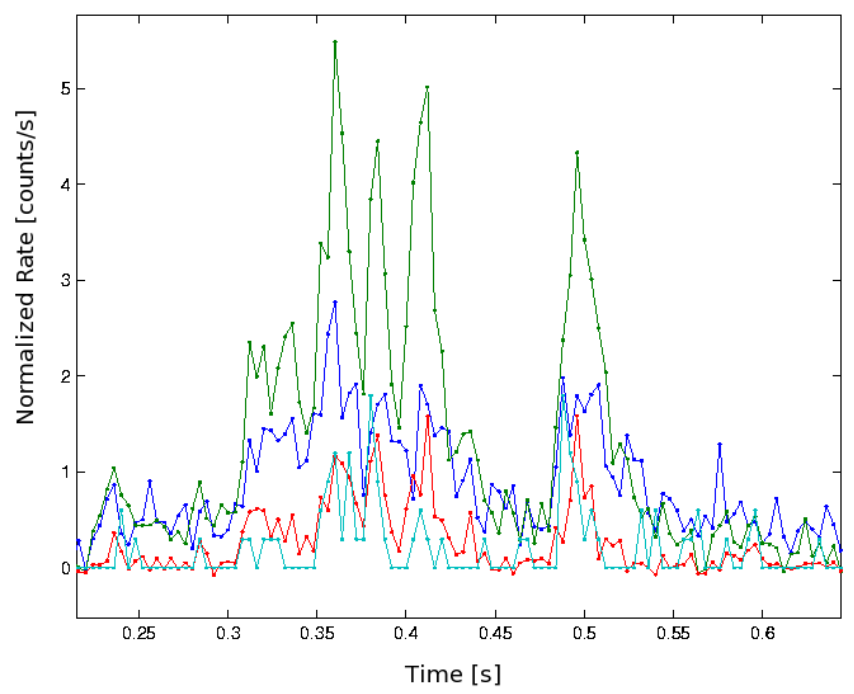

Figure 1: From bottom to top, lights curves of GRB 051221A detected by Swift-BAT in the energy bands 300 - $350 \mathrm{keV}$ (light blue), 100 - $150 \mathrm{keV}$ (red), 15 - $35 \mathrm{keV}$ (dark blue) and 50 - $150 \mathrm{keV}$ (green). The background has been substracted in all the bands except in $300-350 \mathrm{keV}$, where the noise hides the signal otherwise.

\subsection{The Swift-BAT data}

The BAT light curve [10, 12, 13] is depicted at Fig. 1] It shows several bright short peaks with a duration of $\sim 10-15$ msec each. Approximately a second later a second smaller and softer peak followed lasting $\sim 3$ sec.

The overall time resolution of a telescope is determined by the intrinsic detector minimal time resolution, $4 \mathrm{msec}$ for the Swift-BAT data, and the photon arrival rate [8]. For GRB 051221A, the resolution associated with the photon arrival rate in the $\mathrm{keV}$ range is smaller than the instrumental resolution, 4msec. At the higher energy band, (300-350) keV, the photon arrival rate becomes too small and we need to switch to the unfiltered data. Overall, the instrumental resolution is setting the limiting resolution.

To constrain the parameter $\xi$ we compare the light-curves covering the $(15,35),(50,150) \mathrm{keV},(100,150)$ and $(300,350) \mathrm{keV}$ energy bands. We have measured the time positions of the four main peaks in Fig. 1 (measured 
in 4 msec time bins) :

\begin{tabular}{|c|c|c|c|c|}
\hline \multirow{2}{*}{} & \multicolumn{4}{|c|}{ Time position (s) } \\
\cline { 2 - 5 } & 1st peak & 2nd peak & 3rd peak & 4th peak \\
\hline $15-35 \mathrm{keV}$ & 0.360 & 0.388 & 0.408 & 0.496 \\
\hline $50-150 \mathrm{keV}$ & 0.360 & 0.388 & 0.412 & 0.496 \\
\hline $100-150 \mathrm{keV}$ & 0.360 & 0.388 & 0.412 & 0.496 \\
\hline $300-350 \mathrm{keV}$ & 0.360 & 0.380 & 0.408 & 0.488 \\
\hline
\end{tabular}

In the light curve in the $15-35 \mathrm{keV}$ band, the fourth peak is composed of three sub-peaks. The time given in the table corresponds to the middle subpeak. Notice that the first sub-peak is simultaneous with the peak in the 300 $-350 \mathrm{keV}$ band.

At 4 msec time bin resolution we do not appreciate any time delay between the different peaks in all three energy bands. This lack of temporal shift is compared now with the theoretical times delays expected from Eq. 1]

\begin{tabular}{|c|c|c|}
\hline & \multicolumn{2}{|c|}{ Time delay $(\mathrm{s})$} \\
\cline { 2 - 3 } & $n=1$ & $n=2$ \\
\hline$\Delta t_{(15,100) \mathrm{keV}}$ & $4.0 \cdot 10^{-6} / \xi$ & $5.6 \cdot 10^{-29} / \xi^{2}$ \\
\hline$\Delta t_{(100,300) \mathrm{keV}}$ & $9.4 \cdot 10^{-6} / \xi$ & $4.6 \cdot 10^{-28} / \xi^{2}$ \\
\hline$\Delta t_{(15,300) \mathrm{keV}}$ & $1.3 \cdot 10^{-5} / \xi$ & $5.2 \cdot 10^{-28} / \xi^{2}$ \\
\hline
\end{tabular}

As the photon flux decreases with energy, we consider in each energy band the lowest energy as the relevant one for the calculation of $\Delta t$. This conservative estimate lowers slightly the limit we obtain on $\xi$. Since no time delay was observed, we can infer the following bounds:

\begin{tabular}{|c|c|c|}
\hline & $n=1$ & $n=2$ \\
\hline$\Delta t_{(15,100) \mathrm{keV}}$ & $\xi_{1}>0.0010$ & $\xi_{2}>1.2 \cdot 10^{-13}$ \\
\hline$\Delta t_{(100,300) \mathrm{keV}}^{*}$ & $\xi_{1}>0.0023$ & $\xi_{2}>3.4 \cdot 10^{-13}$ \\
\hline$\Delta t_{(15,300) \mathrm{keV}}^{*}$ & $\xi_{1}>0.0033$ & $\xi_{2}>3.6 \cdot 10^{-13}$ \\
\hline
\end{tabular}

* The background signal has not been subtracted in the energy band 300-350 $\mathrm{keV}$ to keep an acceptable photon arrival rate. 


\subsection{The Konus-Wind data}

The Konus-Wind light curve 14 consists of a soft weak precursor and the main episode with five $\sim 15$ msec peaks. The first peak is substantially softer than the others (there is no emission in the 380-1160 keV energy range). After $0.250 \mathrm{sec}$ a weak soft emission is marginally seen only in the $18-70 \mathrm{keV}$ range up to $\sim 1$ sec (see Fig. 2).

The light curve of Konus-Wind is given at 2 msec resolution, corresponding to the intrinsic resolution of the telescope. As in the Swift analysis, the resolution is dictated by the instrumental resolution, even though the inverse photon rate is close to the limiting resolution of the detector. At the high energy band there are only a few photons per temporal bin. The light curve in the 18 - $1160 \mathrm{keV}$ regime has been subdivided in three energy intervals : $18-70 \mathrm{keV}, 70-300 \mathrm{keV}$ and $300-1160 \mathrm{keV}$. The time positions of the four main peaks are :

\begin{tabular}{|c|c|c|c|c|}
\hline & \multicolumn{4}{|c|}{ Time position (s) } \\
\cline { 2 - 5 } & 1st peak & 2nd peak & 3rd peak & 4th peak \\
\hline $18-70 \mathrm{keV}$ & 0.038 & 0.064 & 0.086 & 0.16 \\
\hline $70-300 \mathrm{keV}$ & 0.040 & 0.062 & 0.086 & 0.16 \\
\hline $300-1160 \mathrm{keV}$ & 0.038 & 0.060 & 0.082 & 0.16 \\
\hline
\end{tabular}

The second peak in the energy band $18-70 \mathrm{keV}$ is subdivided into two peaks. We give the position of the second sub-peak where most of the photons were detected.

The expected time delays are:

\begin{tabular}{|c|c|c|}
\hline & \multicolumn{2}{|c|}{ Time delay $(\mathrm{s})$} \\
\cline { 2 - 3 } & $n=1$ & $n=2$ \\
\hline$\Delta t_{(18,70) \mathrm{keV}}$ & $2.4 \cdot 10^{-6} / \xi$ & $2.6 \cdot 10^{-29} / \xi^{2}$ \\
\hline$\Delta t_{(70,300) \mathrm{keV}}$ & $1.0 \cdot 10^{-5} / \xi$ & $4.9 \cdot 10^{-28} / \xi^{2}$ \\
\hline$\Delta t_{(18,300) \mathrm{keV}}$ & $1.3 \cdot 10^{-5} / \xi$ & $5.2 \cdot 10^{-28} / \xi^{2}$ \\
\hline
\end{tabular}

Again, no time delays are observed and, hence, the following bounds emerge 
KONUS - WIND GRB 051221

$\mathrm{T}_{0}=6672.976 \mathrm{~s}$ UT $(01: 51: 12.976)$

S2
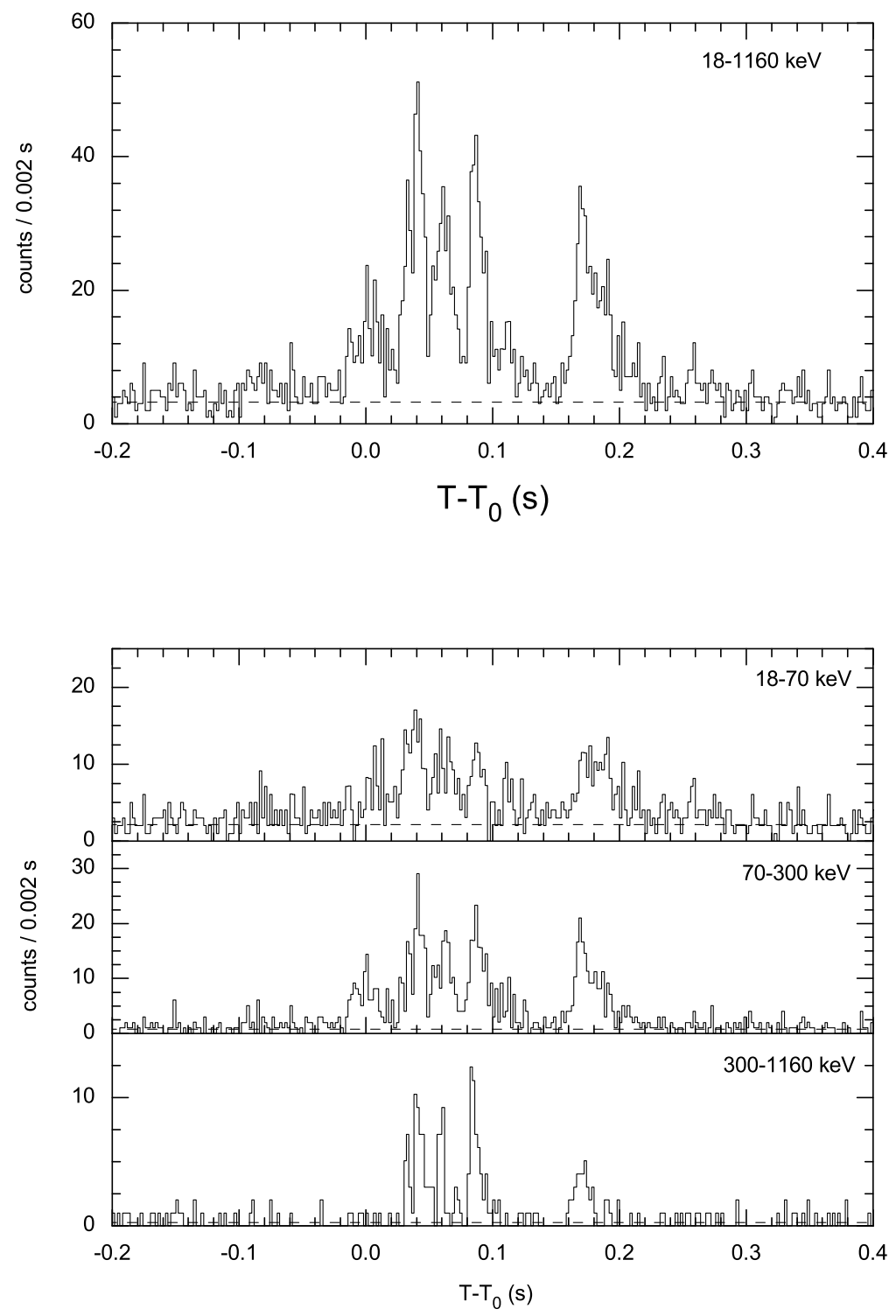

Figure 2: Light curves of GRB 051221A as observed by Konus-Wind 14. From http://www.ioffe.rssi.ru/LEA/GRBs/GRB051221_T06672/. 


\begin{tabular}{|c|c|c|}
\hline & $n=1$ & $n=2$ \\
\hline$\Delta t_{(18,70) \mathrm{keV}}$ & $\xi_{1}>0.0012$ & $\xi_{2}>1.1 \cdot 10^{-13}$ \\
\hline$\Delta t_{(70,300) \mathrm{keV}}$ & $\xi_{1}>0.0054$ & $\xi_{2}>5.0 \cdot 10^{-13}$ \\
\hline$\Delta t_{(18,300) \mathrm{keV}}$ & $\xi_{1}>0.0066$ & $\xi_{2}>5.1 \cdot 10^{-13}$ \\
\hline
\end{tabular}

These bounds are slightly better than the bounds obtained from the Swift data. This improvement is simply due to the better intrinsic time resolution, 2 msec of Konus-Wind versus 4 msec in Swift.

\section{Conclusions}

We set new bounds on the possible energy scale of Lorentz invariance violation. To do so, we used the simultaneity of peak emission times in the light curves in different energy bands observed by Swift-BAT and Konus-Wind for GRB 051221A. The order of magnitude of the bounds depends on how the photon dispersion relation is deformed. In the models where a cubic term in the momentum is added $(n=1)$ the best bound we obtained is $0.0066 E_{p l}$. When a quartic term is added $(n=2)$ the effect is much smaller and the corresponding best bound is $5.1 \cdot 10^{-13} E_{p l}$.

The present work complements previous studies which found bounds of similar order of magnitude. Ellis et al. 4, 5 performed a statistical analysis in an ensemble of bursts with known redshifts (to correct for intrinsic time delays), finding the bounds $\xi_{1}>5.6 \cdot 10^{-4}$ and $\xi_{2}>2.4 \cdot 10^{-13}$ at a $95 \%$ of confidence level. In general, intrinsic delays render difficult to constrain Lorentz violations using a single burst, but we can still extract lower limits if there is simultaneity in the peak emissions. In our study, since no time delays are observed in the light curves with $2 \mathrm{msec}$ bins, we can assume that, at that resolution, all photons were emitted simultaneously. This is, of course, an assumption. We cannot rule out the possibility in which intrinsic spectral temporal shifts are exactly compensated by the time delays produced by the modified dispersion relation, Eq. 1. While, in principle, this is possible, we believe it to be extremely unlikely.

Boggs et al. 6] considered an unique extremely bright burst GRB 021206 observed by RHESSI. This burst has an almost flat spectrum in the band 1 $17 \mathrm{MeV}$, which allowed estimates of the time delay up to $17 \mathrm{MeV}$. The redshift of GRB 021206 is not known as no host galaxy was detected. Assuming a redshift of $z \simeq 0.3$ and using the simultaneity of the peaks at different 
energies, Boggs et al., [6] obtained the bounds $\xi_{1}>0.015$ and $\xi_{2}>4.5 \cdot 10^{-12}$ for $n=1,2$. These lower bounds are higher than ours by factors of 2.5 and 9 respectively. However, the redshift of this burst was not measured, but only estimated from the spectral and temporal properties of the burst. From this point of view, the present estimate, which is based on a burst with a known redshift, is more robust.

\section{Acknowledgments}

We thank Kim Page and the UK Swift Science Data Centre for kindly providing us with the Swift-BAT light curves of GRB 051221A. This research was supported by the EU-RTN "GRBs - Enigma and a Tool", by an US-Israel BSF grant and by the Schwarzmann university chair (TP).

\section{References}

[1] G. Amelino-Camelia, New J. Phys. 6, 188 (2004) arXiv:gr-qc/0212002.

[2] S. R. Coleman and S. L. Glashow, Phys. Rev. D 59, 116008 (1999) arXiv:hep-ph/9812418.

[3] G. Amelino-Camelia, J. R. Ellis, N. E. Mavromatos, D. V. Nanopoulos and S. Sarkar, Nature 393, 763 (1998) arXiv:astro-ph/9712103.

[4] J. R. Ellis, N. E. Mavromatos, D. V. Nanopoulos and A. S. Sakharov, Astron. Astrophys. 402, 409 (2003) arXiv:astro-ph/0210124.

[5] J. Ellis, N. E. Mavromatos, D. V. Nanopoulos, A. S. Sakharov and E. K. G. Sarkisyan, astro-ph/0510172

[6] S. E. Boggs, and C. B. .Wunderer, K. Hurley and W. Coburn, Astrophys. J. 611, L77-L80 (2004) astro-ph/0310307

[7] G., Amelino-Camelia, and T. Piran, T. Phys. Rev D., 64, 036005 (2001)

[8] M. Rodríguez Martínez and T. Piran, arXiv:astro-ph/0601219.

[9] D. N. Spergel et al. [WMAP Collaboration], Astrophys. J. Suppl. 148, 175 (2003) arXiv:astro-ph/0302209. 
[10] A. Parsons et al., GCN 4363.

[11] E. Berger et al., GCN 4384.

[12] J. Cummings et al., GCN 4365.

[13] J. Norris et al., GCN 4388.

[14] S. Golenetskii et al., GCN 4394. 